\title{
Spectral Lower Bounds for the Quantum Chromatic Number of a Graph - Part II
}

\author{
Pawel Wocjan \\ IBM Quantum \\ IBM T.J. Watson Research Center \\ New York, U.S.A. \\ Pawel.Wocjan@ibm.com
}

\author{
Clive Elphick \\ School of Mathematics \\ University of Birmingham \\ Birmingham, U.K. \\ clive.elphick@gmail.com
}

\author{
Parisa Darbari \\ Department of Computer Science \\ University of Central Florida \\ Florida, U.S.A. \\ pdarbar@knights.ucf.edu
}

Submitted: Jan 16, 2020; Accepted: Nov 21, 2020; Published: Dec 11, 2020

(C) The authors. Released under the CC BY license (International 4.0).

\begin{abstract}
Hoffman proved that a graph $G$ with eigenvalues $\mu_{1} \geqslant \cdots \geqslant \mu_{n}$ and chromatic number $\chi(G)$ satisfies:

$$
\chi \geqslant 1+\kappa
$$

where $\kappa$ is the smallest integer such that

$$
\mu_{1}+\sum_{i=1}^{\kappa} \mu_{n+1-i} \leqslant 0 .
$$

We strengthen this well known result by proving that $\chi(G)$ can be replaced by the quantum chromatic number, $\chi_{q}(G)$, where for all graphs $\chi_{q}(G) \leqslant \chi(G)$ and for some graphs $\chi_{q}(G)$ is significantly smaller than $\chi(G)$. We also prove a similar result, and investigate implications of these inequalities for the quantum chromatic number of various classes of graphs, which improves many known results. For example, we demonstrate that the Kneser graph $K G_{p, 2}$ has $\chi_{q}=\chi=p-2$.
\end{abstract}

Mathematics Subject Classifications: 05C15, 05C50 


\section{Introduction}

For any undirected and connected graph $G$ let $V$ denote the set of vertices where $|V|=n$, $A$ denote the adjacency matrix, $\mu_{\max }=\mu_{1} \geqslant \mu_{2} \geqslant \cdots \geqslant \mu_{n}$ denote the eigenvalues of $A$. The multiplicity of the maximum eigenvalue $\mu_{\max }$ is 1 since $G$ is connected. ${ }^{1}$ Let $z$ denote the corresponding (unit) eigenvector.

Let $\chi(G)$ denote the chromatic number and $\omega(G)$ the clique number. Let $\chi_{q}(G)$ denote the quantum chromatic number, as defined by Cameron et al [2]. It is known that $\chi_{q}(G) \leqslant \chi(G)$, and Mancinska and Roberson [11] found a graph on 14 vertices with $\chi(G)>\chi_{q}(G)$, which they suspect is the smallest possible example. There exist graphs for which $\chi_{q}(G)$ is significantly smaller than $\chi(G)$.

Elphick and Wocjan [4] proved that many spectral lower bounds for $\chi(G)$ are also lower bounds for $\chi_{q}(G)$, using linear algebra techniques called pinching and twirling. In this paper, we prove that stronger lower bounds on the chromatic number are also lower bound on the quantum chromatic number.

The following purely combinatorial definition of the quantum chromatic number is due to [11, Definition 1]. For $d>0$, let $I_{d}$ and $0_{d}$ denote the identity and zero matrices in $\mathbb{C}^{d \times d}$. For $c>0$, let $[c]=\{1, \ldots, c\}$.

Definition 1 (Quantum coloring). A quantum $c$-coloring of the graph $G=(V, E)$ is a collection of orthogonal projectors $\left\{P_{v, k}: v \in V, k \in[c]\right\}$ in $\mathbb{C}^{d \times d}$ such that

- for all vertices $v \in V$

$$
\sum_{k \in[c]} P_{v, k}=I_{d} \quad \text { (completeness) }
$$

- for all edges $v w \in E$ and for all $k \in[c]$

$$
P_{v, k} P_{w, k}=0_{d} \quad \text { (orthogonality) }
$$

The quantum chromatic number $\chi_{q}(G)$ is the smallest $c$ for which the graph $G$ admits a quantum $c$-coloring for some dimension $d>0$.

According to the above definition, any classical $c$-coloring can be viewed as a 1dimensional quantum coloring, where we set $P_{v, k}=1$ if vertex $v$ has color $k$ and $P_{v, k}=0$, otherwise. Therefore, quantum coloring is a relaxation of classical coloring. As noted in [11], it is surprising that the quantum chromatic number can be strictly and even exponentially smaller than the chromatic number for certain families of graphs.

We use the following alternative characterization of the quantum chromatic number due to [4]. Before stating this characterization, we briefly review the definition of pinching.

\footnotetext{
${ }^{1}$ The Perron-Frobenius theorem implies that the maximum eigenvalue $\mu_{\max }$ has multiplicity 1 because the adjacency matrix $A$ of the connected graph $G$ is irreducible.
} 
Definition 2 (Pinching). Let $P_{k} \in \mathbb{C}^{m \times m}$ for $k \in[c]$ be orthogonal projectors such that they form a resolution of the identity, that is,

$$
\sum_{k \in[c]} P_{k}=I_{m}
$$

The operation $\mathcal{D}: \mathbb{C}^{m \times m} \rightarrow \mathbb{C}^{m \times m}$ defined by

$$
X \mapsto \mathcal{D}(X)=\sum_{k \in[c]} P_{k} X P_{k}
$$

is called pinching. We say that it annihilates $X$ if $\mathcal{D}(X)=0$.

The following theorem is proved as Theorem 1 in [4]. For the sake of completeness, we include a condensed proof below. We use $\left\{e_{v}: v \in V\right\}$ to denote the standard basis in $\mathbb{C}^{n}$.

Theorem 3. Let $\left\{P_{v, k}: v \in V, k \in[c]\right\}$ be an arbitrary quantum c-coloring of $G$ in $\mathbb{C}^{d}$. Then, the following block-diagonal projectors

$$
P_{k}=\sum_{v \in V} e_{v} e_{v}^{\dagger} \otimes P_{v, k} \in \mathbb{C}^{n \times n} \otimes \mathbb{C}^{d \times d}
$$

define a pinching operation that annihilates $A \otimes I_{d}$, that is,

$$
\sum_{k \in[c]} P_{k}\left(A \otimes I_{d}\right) P_{k}=0
$$

Proof. We have

$$
\begin{aligned}
\sum_{k \in[c]} P_{k} & =\sum_{k \in[c]} \sum_{v \in V} e_{v} e_{v}^{\dagger} \otimes P_{v, k} \\
& =\sum_{v \in V} e_{v} e_{v}^{\dagger} \otimes \sum_{k \in[c]} P_{v, k} \\
& =\sum_{v \in V} e_{v} e_{v}^{\dagger} \otimes I_{d} \\
& =I_{n} \otimes I_{d} .
\end{aligned}
$$

This shows that the orthogonal projectors $P_{k}$ form a resolution of the identity, that is, form a pinching operation.

For $v, w \in V$, let $a_{v w}$ denote the entries of the adjacency matrix. For $k \in[c]$, we have

$$
P_{k}\left(A \otimes I_{d}\right) P_{k}=\sum_{v, w \in V} a_{v w} \cdot e_{v} e_{w}^{\dagger} \otimes P_{v, k} P_{w, k} .
$$

Whenever $a_{v w}=1$, or equivalently $v w \in E$, the corresponding orthogonal projectors $P_{v, k}$ and $P_{w, k}$ must be orthogonal. This shows that the above sum is equal to $0_{d}$, that is, the corresponding pinching operation annihilates $A \otimes I_{d}$. 
Remark 4 . The classical case corresponds simply to the special case $d=1$. In this case, the projectors $P_{k}$ are a diagonal in the standard basis of $\mathbb{C}^{n}$ and each projector corresponds to a color class, that is, each projector $P_{k}$ projects onto the subspace spanned by the standard basis vectors corresponding to the vertices that have been colored with the $k$ th color.

\section{New bounds for the quantum chromatic number}

We use $\uparrow$ to indicate that the eigenvalues are sorted in increasing order. The $i$ th smallest eigenvalue of a hermitian matrix $X \in \mathbb{C}^{n \times n}$ is denoted by $\mu_{i}^{\uparrow}(X)$ so that

$$
\mu_{1}^{\uparrow}(X) \leqslant \mu_{2}^{\uparrow}(X) \leqslant \cdots \leqslant \mu_{n}^{\uparrow}(X) .
$$

Similarly, we use $\downarrow$ to indicate that the eigenvalues are sorted in decreasing order. The $i$ th largest eigenvalue of $X$ is denoted by $\mu_{i}^{\downarrow}(X)$ so that

$$
\mu_{1}^{\downarrow}(X) \geqslant \mu_{2}^{\downarrow}(X) \geqslant \cdots \geqslant \mu_{n}^{\downarrow}(X) .
$$

When the eigenvalues are sorted in decreasing order, we often omit $\downarrow$ so that

$$
\mu_{1}(X) \geqslant \mu_{2}(X) \geqslant \cdots \geqslant \mu_{n}(X)
$$

We have $\mu_{i}^{\uparrow}(X)=\mu_{n+1-i}(X)$. It would be somewhat inconvenient to always have to write $n+i-1$, so this is why the $\uparrow$ notation simplifies the indices.

The following lemma is a standard interlacing result in matrix analysis. We describe it in detail since it is the main result that we rely on to prove the new stronger bounds on the quantum chromatic number.

Lemma 5. Let $X \in \mathbb{C}^{n \times n}$ be a hermitian matrix and let $S \in \mathbb{C}^{n \times m}$ be a matrix such that $S^{\dagger} S=I_{m}$, that is, its $m$ column vectors $s_{1}, \ldots, s_{m}$ are orthonormal vectors. Then,

$$
\mu_{i}^{\uparrow}(X) \leqslant \mu_{i}^{\uparrow}\left(S^{\dagger} X S\right)
$$

for $i \in[m]$.

Proof. This follows from the Courant-Weyl-Fisher theorem stating

$$
\mu_{i}^{\uparrow}(X)=\min _{\mathcal{M}_{i}} \max _{x \in \mathcal{M}_{i}} x^{\dagger} X x
$$

where the minimum is taken over subspaces $\mathcal{M}_{i}$ of dimension $i$ and the maximum is taken over unit vectors $x \in \mathcal{M}_{i}$. By multiplying $X$ by $S^{\dagger}$ and $S$ from the left and right, respectively, we effectively restrict the subspaces $\mathcal{M}_{i}$ to have the form

$$
\mathcal{M}_{i}=\left\{S y: y \in \mathcal{N}_{i}\right\}
$$

where $\mathcal{N}_{i}$ is a subspace of $\mathbb{C}^{m}$ of dimension $i \in[m]$. 
We obtain the following corollary by using the identity $\mu_{i}^{\uparrow}(-X)=-\mu_{i}(X)$ and applying the above lemma to the matrix $-X$.

Corollary 6. Let $X \in \mathbb{C}^{n \times n}$ and $S \in \mathbb{C}^{n \times m}$ with $S^{\dagger} S=I_{m}$ as in Lemma 5. Then

$$
\mu_{i}(X) \geqslant \mu_{i}\left(S^{\dagger} X S\right)
$$

In the following, we will only use this corollary with $i=1$, that is,

$$
\mu_{\max }(X) \geqslant \mu_{\max }\left(S^{\dagger} X S\right) .
$$

\subsection{First new bound for $\chi_{q}(G)$}

Theorem 7 (First bound on quantum chromatic number). Let $\chi_{q}(G)$ be the quantum chromatic number of a connected graph $G$ with adjacency matrix $A$. Let $\kappa$ be the smallest integer such that

$$
0 \geqslant \mu_{\max }(A)+\sum_{i=1}^{\kappa} \mu_{i}^{\uparrow}(A)
$$

holds. Then the quantum chromatic number is bounded from below by

$$
\chi_{q}(G) \geqslant 1+\kappa \text {. }
$$

Proof. Let $\left\{P_{v, k}: v \in V, k \in[c]\right\}$ be any quantum $c$-coloring in dimension $d$. Construct the corresponding collection $\left\{P_{k}: k \in[c]\right\}$ of block-diagonal projectors as in Theorem 3 .

Let $z \in \mathbb{C}^{n}$ denote the unique eigenvector of $A$ corresponding to the largest eigenvalue $\mu_{\max }(A)$. Let $f_{j} \in \mathbb{C}^{d}$ for $j \in[d]$ denote the standard basis vectors. Let $s_{1}, \ldots, s_{m}$ be an orthonormal basis of the subspace

$$
\mathcal{S}=\operatorname{span}\left\{P_{k}\left(z \otimes f_{j}\right): k \in[c], j \in[d]\right\} .
$$

Its dimension $m$ satisfies

$$
d \leqslant m \leqslant c d
$$

For the lower bound, observe that the $d$ orthogonal vectors $z \otimes f_{j}$ are contained in $\mathcal{S}$ since

$$
z \otimes f_{j}=\left(I_{n} \otimes I_{d}\right)\left(z \otimes f_{j}\right)=\left(\sum_{k \in[c]} P_{k}\right)\left(z \otimes f_{j}\right)=\sum_{k \in[c]} P_{k}\left(z \otimes f_{j}\right) .
$$

For the upper bound, observe that there are exactly $c d$ vectors in eq. (22) and, thus, $m$ cannot be larger than $c d$.

Let $S \in \mathbb{C}^{n d \times m}$ be the matrix with $s_{1}, \ldots, s_{m}$ as column vectors. The following two arguments show that the largest eigenvalue of the matrix $S^{\dagger}\left(A \otimes I_{d}\right) S$ is equal to $\mu_{\max }(A)$ and its multiplicity is equal to $d$. First, there exist $d$ orthogonal vectors $y_{1}, \ldots, y_{d} \in \mathbb{C}^{m}$ 
such that $S y_{j}=z \otimes f_{j}$ since the latter vectors are contained in the subspace $\mathcal{S}$, or equivalently, the column space of $S$. We have

$$
\begin{aligned}
S^{\dagger}\left(A \otimes I_{d}\right) S y_{j} & =S^{\dagger}\left(A \otimes I_{d}\right)\left(z \otimes f_{j}\right) \\
& =\mu_{\max }(A) S^{\dagger}\left(z \otimes f_{j}\right) \\
& =\mu_{\max }(A) S^{\dagger} S y_{j} \\
& =\mu_{\max }(A) y_{j} .
\end{aligned}
$$

Second, using Corollary 6 , the largest eigenvalue of $S^{\dagger}\left(A \otimes I_{d}\right) S$ cannot be greater than the largest eigenvalue of $A \otimes I_{d}$.

We can always choose the orthonormal basis vectors $s_{1}, \ldots, s_{m}$ such that for each $i \in[m]$ there exists a unique $k_{i} \in[c]$ with

$$
P_{k_{i}} s_{i}=s_{i} \text { and } P_{k} s_{i}=0 \text { for all } k \neq k_{i} .
$$

This is because $\mathcal{S}=\bigoplus_{k \in[c]} \mathcal{S}_{k}$, where $\mathcal{S}_{k}=\operatorname{span}\left\{P_{k}\left(z \otimes f_{j}\right): j \in[d]\right\}$ since the projectors $P_{k}$ form a resolution of the identity.

We now see that the diagonal entries of the matrix $S^{\dagger}(A \otimes I) S$ must all be zero since

$$
\left(S^{\dagger}(A \otimes I) S\right)_{i i}=s_{i}^{\dagger}\left(A \otimes I_{d}\right) s_{i}=s_{i}^{\dagger} P_{k_{i}}\left(A \otimes I_{d}\right) P_{k_{i}} s_{i}=0 .
$$

For the last equality we used that $P_{k}\left(A \otimes I_{d}\right) P_{k}=0$ for all $k \in[c]$.

So using Lemma 5, we obtain

$$
\begin{aligned}
0 & =\operatorname{tr}\left(S^{\dagger}(A \otimes I) S\right) \\
& =\sum_{i=1}^{m} \mu_{i}^{\uparrow}\left(S^{\dagger}(A \otimes I) S\right) \\
& =\sum_{i=1}^{m-d} \mu_{i}^{\uparrow}\left(S^{\dagger}(A \otimes I) S\right)+d \cdot \mu_{\max }(A) \\
& \geqslant \sum_{i=1}^{m-d} \mu_{i}^{\uparrow}(A \otimes I)+d \cdot \mu_{\max }(A) .
\end{aligned}
$$

Now let $\kappa_{d}$ be the smallest integer such that

$$
0 \geqslant \sum_{i=1}^{\kappa_{d}} \mu_{i}^{\uparrow}(A \otimes I)+d \cdot \mu_{\max }(A) .
$$

Using (23), we have

$$
(c-1) d=c d-d \geqslant m-d \geqslant \kappa_{d} .
$$

Note that $\kappa_{d} \geqslant(\kappa-1) d+1$ must hold because otherwise the condition that $\kappa=\kappa_{1}$ is minimal would be violated. This implies $c-1 \geqslant\left\lceil\kappa_{d} / d\right\rceil=\lceil\kappa-1+1 / d\rceil=\kappa$. In particular, this hold for a quantum $c$-coloring attaining $\chi_{q}(G)$ so that $\chi_{q}(G) \geqslant 1+\kappa$. 
A weaker version of Theorem 7 , with $\chi(G)$ replacing $\chi_{q}(G)$, was proved by Hoffman [10] in 1970. This theorem immediately implies that

$$
1+\frac{\mu_{1}}{\left|\mu_{n}\right|} \leqslant \chi_{q}(G) \leqslant \chi(G)
$$

which was proved in [4] using different techniques.

The proof of the following bound generalises a proof due to Haemers ([7],[8]) from the classical to the quantum chromatic number.

\subsection{Second new bound for $\chi_{q}(G)$}

Theorem 8 (Second bound on quantum chromatic number). For any connected graph $G$ with $\mu_{2}>0$ :

$$
\chi_{q}(G) \geqslant 1+\min \left\{g, \frac{\left|\mu_{n}(A)\right|}{\mu_{2}(A)}\right\},
$$

where $g$ is the multiplicity of $\mu_{n}(A)=\mu_{\min }(A)$.

Proof. Consider an arbitrary quantum $c$-coloring in dimension $d$. Assume that $c \leqslant g$.

Let $\mathcal{S}$ be defined as in the proof of the previous theorem in (22). Let $\mathcal{T}$ be the subspace spanned by the eigenvectors corresponding to the $c d$ smallest eigenvalues $\mu_{1}^{\uparrow}(A \otimes$ $I), \ldots, \mu_{c d}^{\uparrow}(A \otimes I)$. We now show that there exists a non-zero unit vector $y$ with

$$
y \in \mathcal{S}^{\perp} \cap \mathcal{T}
$$

To this end, define $\mathcal{R}=\operatorname{span}\left\{z \otimes f_{j}: j \in[d]\right\}$. Observe that both $\mathcal{S}^{\perp}$ and $\mathcal{T}$ are contained in the subspace $\mathcal{R}^{\perp}$ and

$$
\operatorname{dim} \mathcal{R}^{\perp}=n d-d<n d=(n d-c d)+c d \leqslant \operatorname{dim} \mathcal{T}+\operatorname{dim} \mathcal{S}^{\perp} .
$$

For $i \in[c]$, define $y_{i}=P_{i} y$. Let $m$ be the number of $y_{i}$ that are non-zero. We now show that at least two of them (w.l.o.g. $y_{1}$ and $y_{2}$ ) must be non-zero, that is, $m \geqslant 2$. First of all, at least one must be non-zero because otherwise we would have $0 \neq y=\left(I_{n} \otimes I_{d}\right) y=$ $\left(\sum_{k \in[c]} P_{k}\right) y=\sum_{k \in[c]} y_{k}=0$. Now assume that only $y_{1}$ were non-zero, or equivalently, $y=P_{1} y$. But this leads to the contradiction

$$
0>\mu_{c}^{\uparrow}(A)=\mu_{c d}^{\uparrow}\left(A \otimes I_{d}\right) \geqslant y^{\dagger}\left(A \otimes I_{d}\right) y=y^{\dagger} P_{1}\left(A \otimes I_{d}\right) P_{1} y=0,
$$

where the first inequality holds because $c \leqslant g$, the second inequality holds because $y \in \mathcal{T}$, and the last equality holds because $P_{1}\left(A \otimes I_{d}\right) P_{1}=0$. The latter follows from the fact that $P_{1}$ is one of the projectors forming a pinching that annihilates $A \otimes I_{d}$.

Define the orthonormal vectors $s_{i}=y_{i} /\left\|y_{i}\right\|$ for $i \in[m]$ and $S$ to be the matrix whose columns are $s_{i}$. Define the matrix $X=A \otimes I_{d}-\Delta \cdot z z^{\dagger} \otimes I_{d}$, where $\Delta=\mu_{\max }-\mu_{\min }$ and $z$ is the (unit) eigenvector corresponding to $\mu_{\max }$.

Since $y$ is in the column space of $S$, the smallest eigenvalue of $S^{\dagger} X S$ is at most $y^{\dagger} X y$, which in turn is at most $\mu_{c d}^{\uparrow}\left(A \otimes I_{d}\right)=\mu_{c}^{\uparrow}(A)=\mu_{n}(A)$ as $y \in \mathcal{T}$ and $c \leqslant g$. Also, it holds that $\mu_{\max }\left(S^{\dagger} X S\right) \leqslant \mu_{\max }(X)=\mu_{2}(A)$. 
We now show that the trace of $S^{\dagger} X S$ is equal to 0 . The diagonal entries of $S^{\dagger} X S$ are all zero because

$$
\begin{aligned}
\left(S^{\dagger} X S\right)_{i i} & =s_{i}^{\dagger} X s_{i} \propto y_{i}^{\dagger} X y_{i} \\
& =y^{\dagger} P_{i}\left(A \otimes I_{d}-\Delta \cdot z z^{\dagger} \otimes I_{d}\right) P_{i} y \\
& =y^{\dagger} P_{i}\left(A \otimes I_{d}\right) P_{i} y-\Delta \cdot \sum_{j \in[d]} y^{\dagger} P_{i}\left(z z^{\dagger} \otimes f_{j} f_{j}^{\dagger}\right) P_{i} y \\
& =0
\end{aligned}
$$

where we used that $P_{i}\left(A \otimes I_{d}\right) P_{i}=0$ and $y \perp P_{i}\left(z \otimes f_{j}\right)$ for each $j$. The latter holds as $y \in \mathcal{S}^{\perp}$ and $P_{i}\left(z \otimes f_{j}\right) \in \mathcal{S}$.

Combining that $S^{\dagger} X S$ is traceless with the above bounds on its minimum and maximum eigenvalues yields that

$$
0=\operatorname{tr}\left(S^{\dagger} X S\right)=\sum_{i \in[m]} \mu_{i}^{\uparrow}\left(S^{\dagger} X S\right) \leqslant \mu_{n}(A)+(m-1) \mu_{2}(A)
$$

which completes the proof.

A weaker version of this bound, with $\chi(G)$ replacing $\chi_{q}(G)$, is already known, for example in Corollary 3.6.4 in [1].

We note that both Theorems are also valid for weighted adjacency matrices of the form $W \circ A$, where $W$ is an arbitrary Hermitian matrix and $\circ$ denotes the Hadamard product (also called the Schur product). An example of using a weighted adjacency matrix is to replace $A$ with the normalized adjacency matrix $\mathcal{A}=D^{-1 / 2} A D^{-1 / 2}$, where $D$ is the diagonal matrix of vertex degrees, in both bounds for $\chi_{q}(G)$. This choice of weight matrix reproduces the lower bound for $\chi(G)$ in Theorem 2.2 in Coutinho et al [3], once account is taken of the differences in notation.

\section{Implications for quantum chromatic number}

\subsection{Strongly regular graphs (SRGs)}

Elphick and Wocjan [4] discussed implications of their results for the quantum chromatic number. For example they demonstrated using an inertial bound that the Clebsch graph has $\chi_{q}(G)=4$. Since the Clebsch graph has spectrum $\left(5^{1}, 1^{10},-3^{5}\right)$ this also follows immediately from Theorem 8. The generalised quadrangle, $G Q(2,4)$, on 27 vertices has $\chi=6$, but in [4] the authors were only able to show that $\chi_{q} \geqslant 5$. The spectrum of $G Q(2,4)$ is $\left(10^{1}, 1^{20},-5^{6}\right)$, so from Theorem 8 it follows that $\chi_{q}=6$.

Both of these graphs are strongly regular, and Theorems 7 and 3 can be used to calculate the quantum chromatic number of many strongly regular graphs (SRGs). For example the Kneser graph $K_{p, 2}$ (with $p \geqslant 4$ ) has chromatic number $\chi=p-2$ and spectrum $\left((p-2)(p-3) / 2^{1}, 1^{p(p-3) / 2},(3-p)^{p-1}\right)$, which using Theorem 8 implies $\chi_{q}=\chi=p-2$. 
The Hoffman-Singleton graph, $\operatorname{SRG}(50,7,0,1)$ has $\operatorname{spectrum}\left(7^{1}, 2^{28},-3^{21}\right)$ and $\chi=4$. Theorem 7 implies $\chi_{q}=4$ also.

Fiala and Haemers [5] identified (see their Theorem 10.1) all SRGs with $\chi=5$. So, using Theorem $7, \operatorname{SRG}(15,8,4,4)$ and $\operatorname{SRG}(25,8,3,2)$ have $\chi_{q}=5$; and using Theorem 8 , $\operatorname{SRG}(21,10,3,6)$ and $\operatorname{SRG}(25,16,9,12)$ have $\chi_{q}=5$. The Gewirtz graph, $\operatorname{SRG}(56,10,02)$, has $\chi=4$ and spectrum $\left(10^{1}, 2^{35},-4^{20}\right)$; so using Theorem 7 it has $\chi_{q}=4$.

The Higman-Sims graph is $\operatorname{SRG}(100,22,0,6)$. Its spectrum is equal to $\left(22^{1}, 2^{77},-8^{22}\right)$ and it has $\chi=6$ (see [5]). Theorem 7 implies $\chi_{q} \geqslant 4$ and Theorem 8 implies $\chi_{q} \geqslant 5$. We do not however know whether $\chi_{q}=5$ or 6 . Similarly the $M_{22}$ graph is $\operatorname{SRG}(77,16,04)$ with spectrum $\left(16^{1}, 2^{55},-6^{21}\right)$ has $\chi=5$ (see [5]). Theorems 7 and 8 imply $\chi_{q} \geqslant 4$, but we do now know whether $\chi_{q}=4$ or 5 .

\subsection{Non-SRGs}

The orthogonality graph, $\Omega(n)$, has vertex set the set of \pm 1 -vectors of length $n$, with two vertices adjacent if they are orthogonal. With $4 \mid n$ (see [12]), it is known that $\chi_{q}(\Omega(n))=n$ but $\chi(\Omega(n))$ is exponential in $n$. A proof that $\chi_{q}(\Omega(n))=n$ is as follows. It is immediate from the definition of $\Omega(n)$ that $\xi^{\prime}(\Omega(n))=n$, and it is known that $\chi_{q}(G) \leqslant \xi^{\prime}(G)$, where $\xi^{\prime}(G)$ is the normalized orthogonal rank of $G$ [13]. However, using Theorem 7 and results in section 4.3 of [6] on the eigenvalues of orthogonality graphs we have that:

$$
\chi_{q}(\Omega(n)) \geqslant 1+\frac{\mu_{1}}{\left|\mu_{n}\right|}=1+\frac{1 \cdot 3 \cdots(n-3) \cdot(n-1)}{1 \cdot 3 \cdots(n-3)}=n .
$$

Theorem 9. The orthogonality graph $\Omega(n)$ has a quantum coloring in dimensions pn, where $p$ is a positive integer.

Proof. We can construct a quantum coloring of $\Omega(n)$ using $n$ colors as follows. Let dimension $d=n$, let $U=\operatorname{diag}\left(1, \omega, \ldots, \omega^{n-1}\right)$ be a unitary matrix where $\omega=e^{2 \pi i / n}$, and let $z_{v}$ denote the \pm 1 vector of length $n$ assigned to vertex $v$. Then let

$$
P_{v, k}=U^{k} z_{v} z_{v}^{\dagger}\left(U^{\dagger}\right)^{k}: v \in V, k \in[c] .
$$

It is straightforward that this collection of orthogonal projectors satisfy the completeness and orthogonality conditions in Definition 1, so this completes the quantum coloring with $d=n$.

For $d=p n$, where $p>1$, let

$$
\widetilde{P}_{v, k}=P_{v, k} \otimes I_{p}: v \in V, k \in[c] .
$$

This new collection of orthogonal projectors also satisfy the completeness and orthogonality conditions in Definition 1.

We note in passing that with $4 \mid n$, a proof that $\omega(\Omega(n))=n$, where $\omega(G)$ denotes the clique number of $G$, would provide a proof of the Hadamard Conjecture, which dates from 1867. 
Vertex Transitive(12,27) and Vertex Transitive(12,54) are examples of non-SRGs for which Theorem 8 is exact with $\chi_{q}=4$. Barbell graphs and irregular complete $q$-partite graphs have $\chi_{q}=\chi$, using Theorem 7 .

\subsection{Hoffman colorings}

Any graph for which

$$
1+\frac{\mu_{1}}{\left|\mu_{n}\right|}=\chi(G)
$$

is said to have a Hoffman coloring. All such graphs therefore have $\chi_{q}(G)=\chi(G)$. Examples include SRG $(49,12,5,2)$ which has $\chi_{q}=7$ and the Schlafli graph SRG $(27,16,10,8)$ which has $\chi_{q}=9$. Haemers and Touchev investigated graphs with Hoffman colorings, and Table 1 in [9] lists many such SRGs with up to 100 vertices.

\section{Open questions}

The pentagon $\left(C_{5}\right)$ demonstrates that both of the bounds in this paper are not lower bounds for the vector chromatic number or the fractional chromatic number. The orthogonal rank, $\xi(G)$, is incomparable to $\chi_{q}(G)$. We do not know whether $\xi(G)$ can replace $\chi_{q}(G)$ in Theorems 7 and 8 .

We have shown that the Kneser graph $K_{p, 2}$ has $\chi_{q}=\chi$, but is this true for all Kneser graphs? Are there any strongly regular graphs with $\chi_{q}<\chi$ ?

In Definition 1 the dimension $d$ is any finite positive integer. Let $\chi_{d}(G)$ denote the smallest $c$ for which graph $G$ admits a quantum $c$-coloring in dimension $d$. From Theorem 9, we know that $\chi_{1}(\Omega(n))=\chi(\Omega(n))$ which is exponential in $n$, but $\chi_{p n}(\Omega(n))=n$ for $p$ a positive integer. This raises the question of what is the value of $\chi_{d}(\Omega(n))$ for $d \neq p n$ ? In particular does $\chi_{n+1}(\Omega(n))=n$ ?

\section{Acknowledgement}

We would like to thank David Roberson for insightful comments on an earlier version of this paper.

\section{References}

[1] A. E. Brouwer and W. H. Haemers, Spectra of Graphs, Springer, (2010).

[2] P. J. Cameron, A. Montanaro, M. W. Newman, S. Severini and A. Winter, On the quantum chromatic number of a graph, Electron. J. Combin., 14, (2007), \#R81.

[3] G. Coutinho, R. Grandsire, C. Passsos, Colouring the normalized Laplacian, Elec. Notes in Theoretical Computer Science, 346, (2019), 345 - 354.

[4] C. Elphick and P. Wocjan, Spectral lower bounds for the quantum chromatic number of a graph, J. Combinatorial Theory Ser. A, 168, (2019), 338 - 347. 
[5] N. C. Fiala and W. H. Haemers, 5-chromatic strongly regular graphs, Discrete Math., 306, (2006), 3083 - 3096.

[6] C. Godsil, Interesting graphs and their colourings, (2006).

[7] W. Haemers, Eigenvalue techniques in design and graph theory, Mathematisch Centrum, Amsterdam, (1979).

[8] W. H. Haemers, Interlacing eigenvalues and graphs, Lin. Alg. Appl., 226 - 228, (1995), $593-616$.

[9] W. H. Haemers and V. D. Touchev, Spreads in strongly regular graphs, Designs, Codes and Cryptography, 8, (1996), 145 - 157.

[10] A. J. Hoffman, On eigenvalues and colorings of graphs, in: Graph Theory and its Applications (B. Harris ed.) Acad. Press, New York, (1970).

[11] L. Mančinska and D. E. Roberson, Oddities of Quantum Colorings, Baltic J. Modern Computing, 4, (2016), 846 - 859.

[12] L. Mančinska and D. E. Roberson, Graph homomorphisms for quantum players, J. Combinatorial Theory, Ser. B, 118, (2016), 228 - 267.

[13] P. Wocjan and C. Elphick, Spectral lower bounds for the orthogonal and projective ranks of a graph, Electron. J. Combin., 26(3), (2019), \#P3.45. 